\title{
Chapter 82 \\ Report on the Global Environment \\ Competitiveness of Lebanon
}

Lebanon is located in Southwest and closes to the east of Mediterranean Sea. It borders on Syria and Palestinian. It is tropical Mediterranean climate, and mineral resources is less, mainly is iron, lead, copper and so on. It covers 10 thousand square kilometres. It had a population of 4.2 million and domestic production the gross (GDP)

Score: 47.47

Rank: 94 of USD 40 billion in 2011. Through the evaluation of global environment competitiveness, we can know that the environment competitiveness index of Lebanon ranks at 94 in 133 countries.

Fig. 82.1 Contribution of

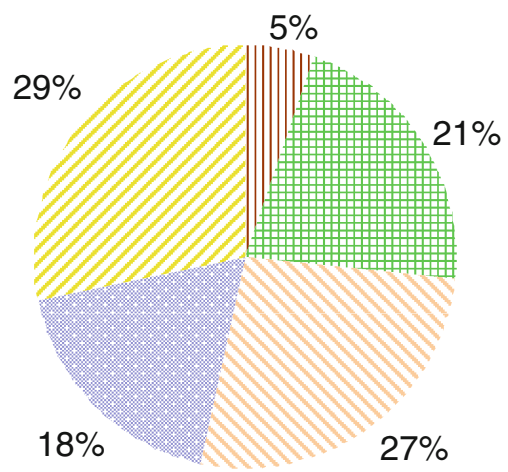

I Resource Environment Competitiveness

\# Ecological Environment Competitiveness Environment Carrying Competitiveness

Environment Management Competitiveness sub-index of GEC Environment Harmony Competitiveness 


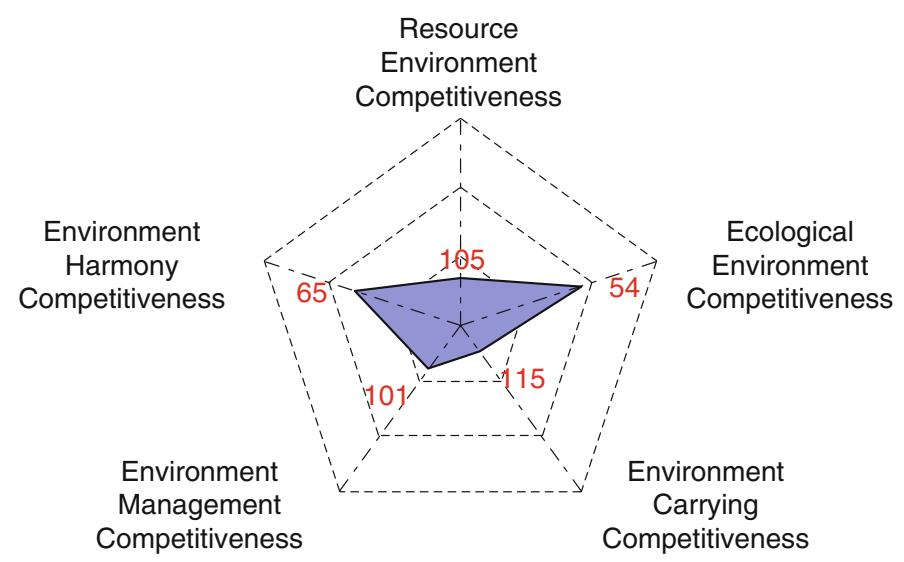

Fig. 82.2 Rank of sub-index of GEC

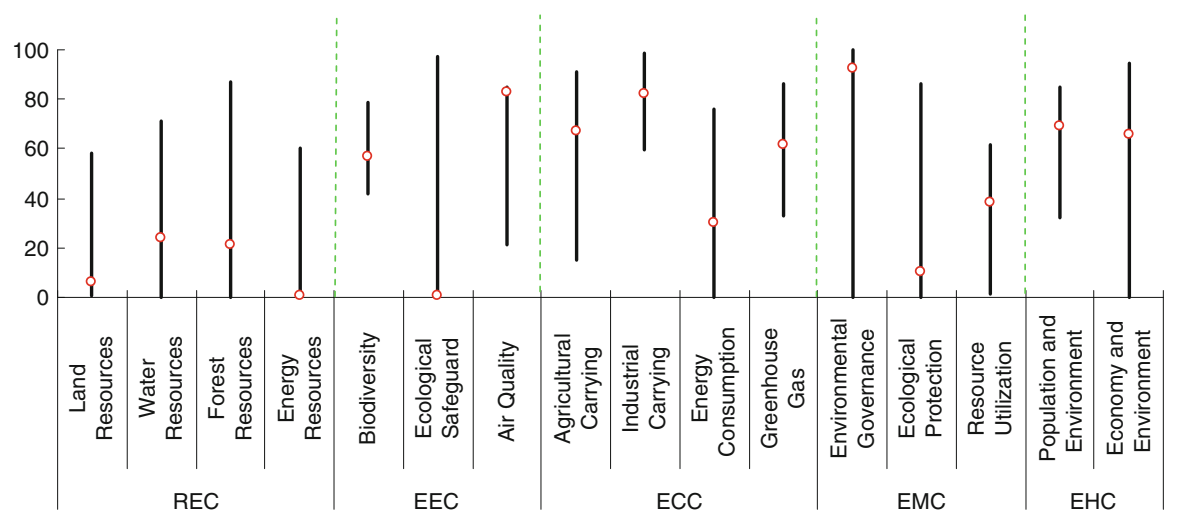

Fig. 82.3 Score and rank of the pillars of GEC

Table 82.1 Score and rank of all indicators of GEC

\begin{tabular}{|c|c|c|c|c|c|}
\hline Indicators & Score & Rank & Indicators & Score & Rank \\
\hline 1 Resource Environment & 12.81 & 105 & Groundwater & 45.03 & 13 \\
\hline Competitiveness & & & \multirow{2}{*}{$\begin{array}{l}\text { Total internal renewable } \\
\text { water resources }\end{array}$} & \multirow[t]{2}{*}{23.66} & \multirow[t]{2}{*}{45} \\
\hline 1.1 Land Resources & 6.06 & 119 & & & \\
\hline Land area per capita & 0.41 & 127 & 1.3 Forest Resources & 21.34 & 89 \\
\hline $\begin{array}{l}\text { Percentage of arable land } \\
\text { to total land area }\end{array}$ & 18.45 & 73 & $\begin{array}{l}\text { Growing stock in forest and } \\
\text { other wooded land }\end{array}$ & 50.02 & 114 \\
\hline Arable land per capita & 1.23 & 128 & \multirow{2}{*}{$\begin{array}{l}\text { Proportion of land area } \\
\text { covered by forest }\end{array}$} & \multirow[t]{2}{*}{15.68} & \multirow[t]{2}{*}{86} \\
\hline 1.2 Water Resources & 23.99 & 44 & & & \\
\hline Surface water & 4.37 & 62 & Forest area per capita & 0.22 & 116 \\
\hline Annual precipitation & 22.89 & 79 & 1.4 Energy Resources & 0.45 & 122 \\
\hline
\end{tabular}


Table 82.1 (continued)

\begin{tabular}{|c|c|c|c|c|c|}
\hline Indicators & Score & Rank & Indicators & Score & Rank \\
\hline Fossil energy & 0.00 & 64 & Energy consumption per unit & 98.66 & 113 \\
\hline Energy production & 0.05 & 123 & of land area & & \\
\hline $\begin{array}{l}\text { Proportion of combustible } \\
\text { renewables and waste to }\end{array}$ & 1.99 & 98 & $\begin{array}{l}\text { Ratio of clean energy } \\
\text { consumption }\end{array}$ & 1.38 & 94 \\
\hline total energy consumption & & & Elasticity of energy & 14.51 & 30 \\
\hline Net energy imports of the & 0.25 & 124 & consumption & & \\
\hline energy consumption & & & Elasticity of electric power & 5.64 & 120 \\
\hline $\begin{array}{l}2 \text { Ecological Environment } \\
\text { Competitiveness }\end{array}$ & 50.38 & 54 & $\begin{array}{l}\text { consumption } \\
\text { 3.4 Greenhouse Gas }\end{array}$ & 61.88 & 64 \\
\hline 2.1 Biodiversity & 56.91 & 82 & Growth rate of $\mathrm{CO}_{2}$ & 66.81 & 24 \\
\hline Threatened fish species & 89.62 & 61 & emissions & & \\
\hline Threatened mammal species & 94.57 & 59 & Growth rate of Methane & 56.49 & 82 \\
\hline Threatened plant species & 99.94 & 22 & emissions & & \\
\hline $\begin{array}{l}\text { GEF benefits index for } \\
\text { biodiversity }\end{array}$ & 0.20 & 109 & $\begin{array}{l}\mathrm{CO}_{2} \text { emissions per unit of } \\
\text { land area }\end{array}$ & 97.98 & 120 \\
\hline 2.2 Ecological Safeguard & 0.65 & 127 & $\mathrm{CO}_{2}$ emissions per unit of & 21.32 & 114 \\
\hline Terrestrial protected areas & 1.09 & 124 & energy consumption & & \\
\hline Marine protected areas & 0.00 & 90 & 4 Environment Management & 43.34 & 101 \\
\hline 2.3 Air Quality & 82.78 & 10 & Competitiveness & & \\
\hline Inhalable particles (PM10) & 81.75 & 55 & 4.1 Environmental & 92.38 & 54 \\
\hline Particulate matter (PM2.5) & 100.00 & 1 & & & \\
\hline Index of indoor air pollution & 100.00 & 1 & $\begin{array}{l}\text { Agricultural chemicals } \\
\text { regulation }\end{array}$ & 80.95 & 53 \\
\hline Nitrogen oxides emission & 68.87 & 16 & Percentage of the rural & 100.00 & 1 \\
\hline Sulfur dioxide emission & 40.68 & 65 & population with access & & \\
\hline $\begin{array}{l}3 \text { Environment Carrying } \\
\text { Competitiveness }\end{array}$ & 63.24 & 115 & $\begin{array}{l}\text { to an improved water } \\
\text { source }\end{array}$ & & \\
\hline 3.1 Agricultural Carrying & 67.02 & 62 & Percentage of the urban & 100.00 & 1 \\
\hline $\begin{array}{l}\text { Cereal yield per unit of } \\
\text { arable land }\end{array}$ & 26.72 & 69 & $\begin{array}{l}\text { population with access } \\
\text { to an improved water }\end{array}$ & & \\
\hline Fertilizer consumption per & 98.39 & 36 & source & & \\
\hline unit of arable land & & & 4.2 Ecological Protection & 10.52 & 122 \\
\hline $\begin{array}{l}\text { Annual freshwater } \\
\text { withdrawals for }\end{array}$ & 89.36 & 112 & $\begin{array}{l}\text { Area of plantation and } \\
\text { afforestation }\end{array}$ & 0.01 & 111 \\
\hline agriculture per unit of & & & Biome protect & 2.80 & 127 \\
\hline $\begin{array}{l}\text { arable land } \\
\text { 3.2 Industrial Carrying }\end{array}$ & 82.49 & 116 & $\begin{array}{l}\text { Overfishing of fishing } \\
\text { resources }\end{array}$ & 32.26 & 87 \\
\hline $\begin{array}{l}\text { Net exports as a percentage } \\
\text { of GDP }\end{array}$ & 93.13 & 10 & 4.3 Resource Utilization & 38.06 & 62 \\
\hline Electric power consumption & 45.95 & 125 & $\begin{array}{l}\text { Utilization rate of water } \\
\text { resources }\end{array}$ & 1.14 & 32 \\
\hline $\begin{array}{l}\text { per unit of value added } \\
\text { of industry }\end{array}$ & & & $\begin{array}{l}\text { Percentage of total internal } \\
\text { renewable water }\end{array}$ & 65.41 & 65 \\
\hline $\begin{array}{l}\mathrm{SO}_{2} \text { emissions per unit of } \\
\text { value added of industry }\end{array}$ & 98.55 & 124 & $\begin{array}{l}\text { resources to total water } \\
\text { resources }\end{array}$ & & \\
\hline $\begin{array}{l}\text { Annual freshwater } \\
\text { withdrawals for industry }\end{array}$ & 92.31 & 106 & $\begin{array}{c}\text { Percentage of agricultural } \\
\text { land to total land area }\end{array}$ & 79.49 & 19 \\
\hline $\begin{array}{l}\text { per value added of } \\
\text { industry }\end{array}$ & & & $\begin{array}{l}\text { Percentage of fossil fuel } \\
\text { energy consumption to }\end{array}$ & 6.21 & 106 \\
\hline 3.3 Energy Consumption & 30.05 & 121 & total energy consumption & & \\
\hline
\end{tabular}


Table 82.1 (continued)

\begin{tabular}{|c|c|c|c|c|c|}
\hline Indicators & Score & Rank & Indicators & Score & Rank \\
\hline $\begin{array}{l}5 \text { Environment Harmony } \\
\text { Competitiveness }\end{array}$ & 67.57 & 65 & $\begin{array}{l}\mathrm{CO}_{2} \text { emissions (metric tons } \\
\text { per capita) }\end{array}$ & 88.16 & 81 \\
\hline $\begin{array}{l}\text { 5.1 Population and } \\
\text { Environment }\end{array}$ & 69.10 & 74 & $\begin{array}{l}\text { Energy consumption per } \\
\text { capita }\end{array}$ & 89.06 & 66 \\
\hline \multirow{2}{*}{$\begin{array}{l}\text { Improved sanitation facilities } \\
\text { (\% of population with } \\
\text { access) }\end{array}$} & 98.00 & 33 & $\begin{array}{l}\text { 5.2 Economy and } \\
\text { Environment }\end{array}$ & 66.04 & 58 \\
\hline & 46.67 & 102 & $\begin{array}{l}\text { Land resource utilization } \\
\text { efficiency }\end{array}$ & 1.14 & 19 \\
\hline $\begin{array}{l}\text { Motor vehicles (per 1,000 } \\
\text { people) }\end{array}$ & 1.36 & 99 & $\begin{array}{l}\text { Sulfur dioxide emissions } \\
\text { per unit of GDP }\end{array}$ & 90.70 & 85 \\
\hline $\begin{array}{l}\text { Renewable internal } \\
\text { freshwater resources } \\
\text { per capita }\end{array}$ & & & $\begin{array}{l}\text { Carbon dioxide emissions } \\
\text { per unit of GDP }\end{array}$ & 82.48 & 84 \\
\hline $\begin{array}{l}\mathrm{SO}_{2} \text { emissions (metric tons } \\
\text { per capita) }\end{array}$ & 89.19 & 98 & $\begin{array}{l}\text { Energy consumption per unit } \\
\text { of GDP }\end{array}$ & 89.85 & 44 \\
\hline
\end{tabular}

Table 82.2 Rank distribution of the individual indicators of GEC

\begin{tabular}{lllllll}
\hline & $\begin{array}{l}\text { Number } \\
\text { of the } \\
\text { individual } \\
\text { indicators }\end{array}$ & $\begin{array}{l}\text { Rank } \\
1-10\end{array}$ & $\begin{array}{l}\text { Rank } \\
11-30\end{array}$ & $\begin{array}{l}\text { Rank } \\
31-60\end{array}$ & $\begin{array}{l}\text { Rank } \\
61-100\end{array}$ & $\begin{array}{l}\text { Rank } \\
101-133\end{array}$ \\
\hline Sub-index & 14 & 0 & 1 & 1 & 7 & 5 \\
\hline $\begin{array}{c}\text { Resource Environment } \\
\quad \text { Competitiveness } \\
\text { Ecological Environment } \\
\quad \text { Competitiveness }\end{array}$ & 11 & 3 & 2 & 2 & 2 & 2 \\
$\begin{array}{c}\text { Environment Carrying } \\
\quad \text { Competitiveness }\end{array}$ & 15 & 1 & 2 & 1 & 5 & 6 \\
$\begin{array}{c}\text { Environment Management } \\
\quad \text { Competitiveness }\end{array}$ & 10 & 1 & 1 & 3 & 2 & 3 \\
$\begin{array}{c}\text { Environment Harmony } \\
\text { Competitiveness }\end{array}$ & 10 & 0 & 1 & 2 & 6 & 1 \\
Total & 60 & 5 & 7 & 9 & 22 & 17 \\
\hline
\end{tabular}

Open Access This chapter is distributed under the terms of the Creative Commons Attribution Noncommercial License, which permits any noncommercial use, distribution, and reproduction in any medium, provided the original author(s) and source are credited. 